Non-standard solar models to resolve the solar neutrino problem fail tests imposed by helioseismology, suggesting that such models are rather contrived, with resolution of the neutrino problem lying in the realm of particle physics.

\title{
Using Helioseismology to Constrain Solar Models
}

The energy radiated by the Sun derives from the fusion of four hydrogen atoms into one helium atom via different paths. The reaction generates approximately $4.1 \mathrm{x}$ $10^{-12} \mathrm{~J}$ (the precise amount depends on the energy lost as neutrinos) and since the Earth receives about $1360 \mathrm{Wm}^{-2}$ from the Sun, the total flux of neutrinos must be around $7 \times 10^{14} \mathrm{~m}^{-2} \mathrm{~s}^{-1}$. The cross-section for neutrino absorption is so small that virtually all the neutrinos escape immediately from the Sun, in contrast to the radiative energy which reaches the solar surface through a slow diffusive process since the mean-free path of a photon near the solar centre is only a few mm. Thus, the neutrinos in principle provide direct information about nuclear reactions in the solar core.

Calculating an accurate estimate of the neutrino detection rate is not trivial for it relies on models that follow numerically the Sun's evolution during the conversion of hydrogen into helium (Fig. 1 gives the dominant reaction paths). Results depend on nuclear reaction rates and on the assumed physics of matter, and involve simplifying assumptions, the most important probably being to neglect hydrodynamic processes that may result in a partial mixing. The outcome is called a "standard" solar model and analogous calculations for other stars form the basis for many areas of astrophysics. Clearly, any discrepancy between the observed and the predicted rates of solar neutrino detection casts doubt on this basis.

The initial helium abundance, which cannot be determined reliably from spectroscopic observations, is treated as a free parameter, being adjusted until the computed luminosity agrees with observations. The computations also involve a second a priori unknown parameter describing convective heat transport near the solar surface; its value is fixed by requiring the model to give the correct radius for the Sun.

The rate of neutrino detection depends strongly on the neutrino energy. In standard solar models, about $85 \%$ of the energy production comes from the PP-I chain (see Fig. 1), with the PP-III chain contributing

Jorgen Christensen-Dalsgaard has been an Associate Professor in the Institute of Physics and Astronomy, Aarhus University, DK-8000 Aarhus C, since nomy, Aarhus University, DK-8000 Aarhus C, since
1984 and was appointed this year to be the Associate Director of the Theoretical Astrophysics Center Aarhus. After graduating from Aarhus University in 1975 , he received his $\mathrm{PhD}$ from Cambridge University in 1978 and then held postdoctoral positions at the University of Liege, the National Center for Atmospheric Research, Boulder, CO (USA), and NORDITA, Copenhagen. Professor Christensen-Dals gaard participates in the Global Oscillations Network Group and is a Co-investigator on the SOI and GOLF instruments to be launched aboard $\mathrm{SOHO}$.

\section{Jørgen Christensen-Dalsgaard}

Teoretisk Astrofysik Center, Danmarks Grundforskningfond Institut for Fysik og Astronomi, Aarhus Universitet

\begin{tabular}{clll}
\hline \multicolumn{1}{c}{ PP-I } & \multicolumn{1}{c}{ PP-II } & \multicolumn{1}{c}{ PP-III } \\
${ }^{1} \mathrm{H}+{ }^{1} \mathrm{H} \rightarrow{ }^{2} \mathrm{D}+\mathrm{e}^{+}+\nu_{\mathrm{e}}$ & ${ }^{3} \mathrm{He}+{ }^{4} \mathrm{He} \rightarrow{ }^{7} \mathrm{Be}+\gamma$ & ${ }^{7} \mathrm{Be}+{ }^{1} \mathrm{H} \rightarrow{ }^{8} \mathrm{~B}+\gamma$ \\
${ }^{2} \mathrm{D}+{ }^{1} \mathrm{H} \rightarrow{ }^{3} \mathrm{He}+\gamma$ & ${ }^{7} \mathrm{Be}+\mathrm{e}^{-} \rightarrow{ }^{7} \mathrm{Li}+\nu_{\mathrm{e}}$ & ${ }^{8} \mathrm{Be}$ & $\rightarrow{ }^{8} \mathrm{~B}+\mathrm{e}^{+}+\nu_{\mathrm{e}}$ \\
${ }^{3} \mathrm{He}+{ }^{3} \mathrm{He} \rightarrow{ }^{4} \mathrm{He}+2^{1} \mathrm{H}$ & ${ }^{7} \mathrm{Li}+{ }^{1} \mathrm{H} \rightarrow{ }^{4} \mathrm{He}+{ }^{4} \mathrm{He}$ & ${ }^{8} \mathrm{Be}$ & $\rightarrow{ }^{4} \mathrm{He}+{ }^{4} \mathrm{He}$ \\
\hline
\end{tabular}

Fig. 1 - The most important hydrogen-burning reactions in the Sun, the so-called PP-I, PP-II and PP-III chains. The initial reaction is the fusion of two hydrogen nuclei into a deuterium nucleus; charge and lepton number conservation demands emission of a positron and a neutrino. This reaction controls the overall rate of energy production. The subsequent reaction path depends on the branchings between the ${ }^{3} \mathrm{He}+{ }^{3} \mathrm{He}$ and the ${ }^{3} \mathrm{He}+{ }^{4} \mathrm{He}$ reactions, and between the ${ }^{7} \mathrm{Be}+e^{-}$and the ${ }^{7} \mathrm{Be}+{ }^{1} \mathrm{H}$ reactions. The PP-III branch is very rare. Neutrinos produced in the ${ }^{1} \mathrm{H}+{ }^{1} \mathrm{H}$ reaction, the electron capture in ${ }^{7} \mathrm{Be}$, and the positron decay of ${ }^{8} \mathrm{~B}$, have average energies of $0.26 \mathrm{MeV}, 1.06 \mathrm{MeV}$ and $7.46 \mathrm{MeV}$, respectively. Neutrinos are also emitted by the rare triple reaction ${ }^{1} \mathrm{H}\left({ }^{1} \mathrm{He}^{-}, \nu_{e}\right)^{2} \mathrm{D}$, and modest contributions to the energy generation and the neutrino flux come from hydrogen fusion through the so-called CNO-cycle.

less than $0.02 \%$. So the constraint that the computed luminosity should fit the observed value essentially determines the number of low-energy neutrinos from the ${ }^{1} \mathrm{H}\left({ }^{1} \mathrm{H}, \mathrm{e}^{+} \nu_{\mathrm{e}}\right)^{2} \mathrm{D}$ reaction. In contrast, the flux of high-energy neutrinos, especially that for the ${ }^{8} \mathrm{~B}\left(, \mathrm{e}^{+} \nu_{\mathrm{e}}\right)^{8} \mathrm{Be}$ reaction, depends on the branching ratios between the different chains which are in turn strongly temperature-dependent, with the fraction of the reactions that go through the PP-II and PP-III chains increasing very rapidly with temperature; so therefore does the flux of high-energy neutrinos. The table overleaf presents rates for a standard model computed using representative and up-to-date values for the various variables. The number of detections is conventionally given in Solar Neutrino Units (SNU), where one SNU corresponds to $10^{-36}$ captures per target atom per second.
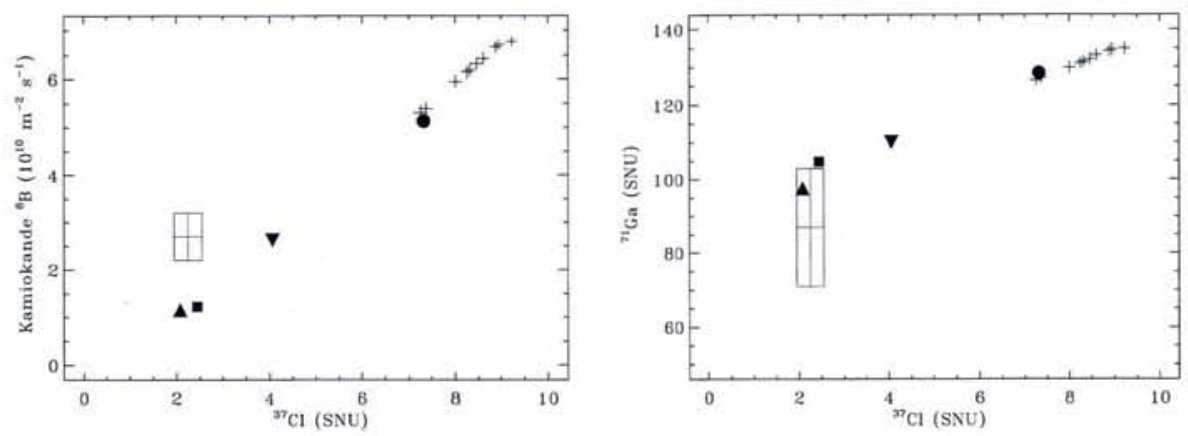

Fig. 2 - Measured and computed neutrino event rates for the Kamiokande (left) and Ga (right) experiments. The measured rates are indicated by error boxes and the large filled circles and squares show the calculated results of Table 1, where the filled squares include the effect of WIMPs simulated through a reduction in the core opacity. The crosses are based on other standard models, differing in equation of state, opacity tables, nuclear parameters, or age. The filled triangles pointing down and up are for partially and fully mixed models, respectively. 
One cannot exclude a "boring" solution to the neutrino problem involving modification of, for example, opacities and the branching ratios in the PP chains. In particular, since the gallium experiments seem consistent with the neutrinos from the reaction which dominates the energy generation, one may in principle be able to reduce the capture rate while maintaining the solar surface luminosity. Further measurements of the relevant nuclear reactions, particularly the somewhat uncertain ${ }^{7} \mathrm{Be}\left({ }^{1} \mathrm{H}, \gamma\right){ }^{8} \mathrm{~B}$ reaction, are required. The possibility of remaining experimental problems must also be taken into account [4].

\section{Non-standard models}

Most attempts to make the predicted neutrino capture rate agree with observations involve changes in the temperature distribution in the solar core to reduce the central temperature, and hence the flux of highenergy neutrinos, while retaining the total energy production. The temperature gradient in the solar interior must be sufficient to ensure transport of the energy from the core to the surface. Therefore, if the effective conductivity of the solar interior is

\section{MEASUREMENTS OF THE SOLAR NEUTRINO FLUX}

Owing to the small neutrino cross-section, measuring the flux of solar neutrinos requires carefully designed, large detectors. The first experiment used the reaction $\nu_{\mathrm{e}}+{ }^{37} \mathrm{Cl} \rightarrow \mathrm{e}^{-}+{ }^{37} \mathrm{Ar}$ with a detector consisting of a tank containing about 380000 litres of $\mathrm{C}_{2} \mathrm{Cl}_{4}$ (a common cleaning fluid) deeply buried in the Homestake mine in South Dakota, USA, to reduce the background from other reactions. Despite the large quantity of detector material, a solar neutrino event is recorded on average only once every second day; the average of the measured capture rates over almost 20 years gives an observed capture rate of $2.2 \pm 0.2 \mathrm{SNU}$. From the table we see that the dominant contribution to ${ }^{37} \mathrm{Cl}$ observations comes from the ${ }^{8} \mathrm{~B}$ neutrinos, despite the relatively low flux, and that the computed value of about 7 SNU does not agree with experiment.

The Kamiokande II experiment has measured the scattering of neutrinos on electrons. It is located in a mine in Japan and consists of a tank containing 3000 tonnes of water, of which 680 tonnes are used for neutrino detection. This experiment records the direction of the neutrinos, confirming that they do indeed come from the Sun; it is exclusively sensitive

increased, the temperature gradient, and hence the neutrino flux, is reduced. This can be accomplished through an overall reduction of the opacity, e.g., by reducing the abundance of elements heavier than $\mathrm{H}_{2}$ and $\mathrm{He}$. Alternatively, the efficiency of energy transport can be increased by invoking other modes of transport.

One suggestion is that the solar core contains hypothetical weakly interacting massive particles (WIMPS) which transport a significant fraction of the luminous output in the solar core. I have simulated their presence through a localized reduction in the opacity in the solar core to give the neutrino rates shown in the table and Fig. 2. It is evident that the ${ }^{37} \mathrm{Cl}$ and the ${ }^{71} \mathrm{Ga}$ capture rates are consistent with the measurements. Note, however, that for the ${ }^{37} \mathrm{Cl}$ experiment the contribution from ${ }^{8} \mathrm{~B}$ is reduced much more strongly than the contribution from ${ }^{7} \mathrm{Be}$. As a result, the predicted rate for the Kamiokande experiment which only sees neutrinos from ${ }^{8} \mathrm{~B}$ is now too low. This is a general problem in attempts to match simultaneously the ${ }^{37} \mathrm{Cl}$ and Kamiokande measurements through modifying solar models [3].

to the ${ }^{8} \mathrm{~B}$ neutrinos. The observed rate of neutrino events corresponds to a flux of ${ }^{8} \mathrm{~B}$ neutrinos of about $(2.7 \pm 0.5) \times 10^{10}$ $\mathrm{m}^{-2} \mathrm{~s}^{-1}$, roughly half the theoretical value (see table).

Measurements sensitive to the neutrinos from the basic ${ }^{1} \mathrm{H}+{ }^{1} \mathrm{H}$ reaction can be obtained from the reaction $\nu_{\mathrm{e}}+{ }^{71} \mathrm{Ga} \rightarrow \mathrm{e}^{-}$ $+{ }^{71} \mathrm{Ge}$ which can detect neutrinos of energy exceeding $0.23 \mathrm{MeV}$. Two such experiments have been set up: the SAGE collaboration which uses a detector currently consisting of almost 60 tonnes of metallic gallium, situated in southern Russia; and the GALLEX experiment using 30 tonnes of gallium in the form of a gallium chloride solution, located in a tunnel under the Gran Sasso massive in Italy. The experiments have given initial results over the last two years, which appear to be consistent. In particular, an average over several runs of the GALLEX experiment yields a capture rate of $87 \pm 16 \mathrm{SNU}$. This is still below the predicted value of 128 SNU (see table), although by a smaller margin than for the ${ }^{37} \mathrm{Cl}$ and electron scattering experiments. Indeed, the table shows that the rate of detection is roughly consistent with the neutrino production from the $\mathrm{pp}$ reactions.

Predicted neutrino fluxes and capture rates in ${ }^{37} \mathrm{Cl}$ and ${ }^{71} \mathrm{Ga}$ detectors for a standard solar model and for a model where the opacity has been reduced near the centre of the Sun.

\begin{tabular}{|c|c|c|c|c|c|c|}
\hline \multirow{4}{*}{$\begin{array}{l}\text { Neutrino } \\
\text { source }\end{array}$} & \multicolumn{3}{|c|}{ Standard model } & \multicolumn{3}{|c|}{ Reduced temperature } \\
\hline & \multirow{3}{*}{$\begin{array}{c}\text { Flux } \\
10^{14} \mathrm{~m}^{-2} \mathrm{~s}^{-1}\end{array}$} & \multirow{2}{*}{\multicolumn{2}{|c|}{$\begin{array}{c}\text { Capture rate } \\
\text { SNU }\end{array}$}} & \multirow{3}{*}{$\begin{array}{c}\text { Flux } \\
10^{14} \mathrm{~m}^{-2} \mathrm{~s}^{-1}\end{array}$} & \multirow{2}{*}{\multicolumn{2}{|c|}{$\begin{array}{c}\text { Capture rate } \\
\text { SNU }\end{array}$}} \\
\hline & & & & & & \\
\hline & & ${ }^{37} \mathrm{Cl}$ & ${ }^{71} \mathrm{Ga}$ & & ${ }^{37} \mathrm{Cl}$ & ${ }^{71} \mathrm{Ga}$ \\
\hline $\mathrm{pp}$ & $6.01 \times 10^{0}$ & 0.00 & 70.9 & $6.23 \times 10^{0}$ & 0.00 & 73.5 \\
\hline pep & $1.43 \times 10^{-2}$ & 0.23 & 3.1 & $1.70 \times 10^{-2}$ & 0.27 & 3.7 \\
\hline${ }^{7} \mathrm{Be}$ & $4.65 \times 10^{-1}$ & 1.12 & 34.0 & $3.03 \times 10^{-1}$ & 0.73 & 22.2 \\
\hline${ }^{8} \mathrm{~B}$ & $5.13 \times 10^{-4}$ & 5.59 & 12.5 & $1.22 \times 10^{-1}$ & 1.33 & 3.0 \\
\hline $\mathrm{CNO}$ & $4.53 \times 10^{-2}$ & 0.38 & 8.1 & $1.42 \times 10^{-2}$ & 0.12 & 2.5 \\
\hline Total: & & 7.32 & 128.5 & & 2.45 & 104.9 \\
\hline
\end{tabular}

Mixing might bring additional hydrogen to the solar core, allowing the solar luminosity to be generated at lower temperature and hence reducing the predicted neutrino capture rates. Fig. 1 also shows results for models with partial and complete mixing of the solar interior; only the latter gives a predicted rate for the ${ }^{37} \mathrm{Cl}$ experiment in accordance with observations.

\section{Conclusions}

In conclusion, some experimental results are disturbing, notably a measured flux which is smaller than the predicted value, in some cases by factors of 2-3. Whereas initial experiments were only sensitive to high-energy neutrinos coming from rare reactions that contribute little to the energy generation and have rates which depend very strongly on the temperature of the solar core, it has been difficult to achieve better agreement via minor adjustments to the model's parameters given that the observed solar luminosity should be reproduced. Although the discrepancy is less marked, the observed detection rate is once again less than the predicted rate for the more recent experiments which detect neutrinos coming from the principal energy-generating reactions. In general, attempts to solve the neutrino problem by adjusting the solar standard model are essentially ad hoc since they have little independent justification beyond the phenomenon being explained. Hence the importance of helioseismic data which may constrain models of the core [5] and thereby test for departures from standard evolution theory.

\section{Solar Oscillations}

The Sun pulsates in many independent modes of oscillation, with periods of about five minutes. The frequencies, which can be determined very precisely, depend on the structure of the solar interior. Some of the modes penetrate virtually to the solar centre so suitable combinations of frequencies give information about the solar core.

Although a variety of observational techniques have been used to study the oscillations of the Sun, the most extensive data come from measurements of the line-ofsight velocity of the solar surface using the Doppler shift of lines in the radiative spectrum. Fig. 3 gives an example of a power spectrum resulting from such observations, in light integrated over the solar surface. Each oscillation mode depends on the position on the solar surface as a spherical harmonic $Y_{,}^{m}(\theta, \varphi)$ of degree $/$ and azimuthal order $m$ with $|m| \leq l$, where $\theta$ is the angular distance from the pole and $\varphi$ is the longitude. A mode is also described by its radial order $n$ corresponding in most cases to the number of nodes in the radial direction.

Examples of spherical harmonics are shown on the front cover. The degree I measures, roughly speaking, the complexity of the mode on the solar surface, whereas $m$ is one-half the number of nodes in longitude. In observations in integrated light, such as those shown in Fig. 3, the contributions from the higher I are largely suppressed due to cancellation between regions of positive and negative velocity. Hence, Fig. 3 is entirely dominated by 
Fig. 3 - The power spectrum of solar oscillations obtained from Doppler observations in light integrated over the disk of the Sun. The ordinate is normalized to show velocity power per frequency bin. The data were obtained by the Birmingham group from two observing stations, on Hawaii and Tenerife, and span 53 days [2]. Note the organization of the spectrum into uniformly spaced pairs of peaks (an example is indicated). Within each pair, the frequencies differ by a small amount $\delta \nu$ which gives information about the properties of the solar core (see text).

modes with $I \leq 3$. The frequencies depend principally on $n$ and $l$; departures from spherical symmetry, such as rotation, introduce a dependence on $m$, but this can be neglected in probing the overall structure of the solar interior.

The individual spherical harmonics can be separated by spatially transforming observations of the velocity field as a function of $\theta$ and $\varphi$. The individual modes are then isolated by carrying out a Fourier transform in time, leading to a power spectrum such as the one shown in Fig. 3. Solar oscillations contain modes of all degrees from zero to about 2000, the distribution of power with frequency and the mean amplitude per mode being roughly independent of $/$ and $m$ and hence corresponding to Fig. 3. Finally, the cyclic frequencies $\nu_{n /}$ of the modes are determined through suitable fitting to the power spectrum.

\section{Comparing observations with predictions}

The value of the frequencies as diagnostics of the solar interior lies in the fact that they can be determined experimentally with high precision, owing to the very long lifetime of at least some of the modes. An indication of this is the sharpness of the lines in the spectrum of Fig. 3 . The most precisely observed frequencies have been determined with a relative error of less than $10^{-5}$ The observations can be compared with frequencies computed quite accurately using solar models. As indicated above, given the physical properties in the Sun (including the equation of state, the absorption coefficient for radiation, and nuclear reaction rates) one first solves the equations of stellar structure and evolution by following the change with time of the chemical composition. The outcome is a table giving the variation of say pressure, density, temperature, and speed of sound with position. One then solves the equations for small-amplitude stellar oscillations obtained by linearizing the equations of hydrodynamics. These equations, and the boundary conditions, define the oscillations as eigenfrquencies in the same way as the energy levels of a hydrogen atom. The energetics of the oscillations have no effect on the computed frequencies, so the physical properties of the oscillations are well established except close to the solar surface, where energy exchange and interaction with the turbulent convection affects frequencies in a manner that is difficult to model (although any uncertainties can be largely eliminated in the detailed analysis of frequencies).

\section{The nature of oscillations}

To understand the diagnostic capabilities of the frequencies requires some insight into creasing 1 .

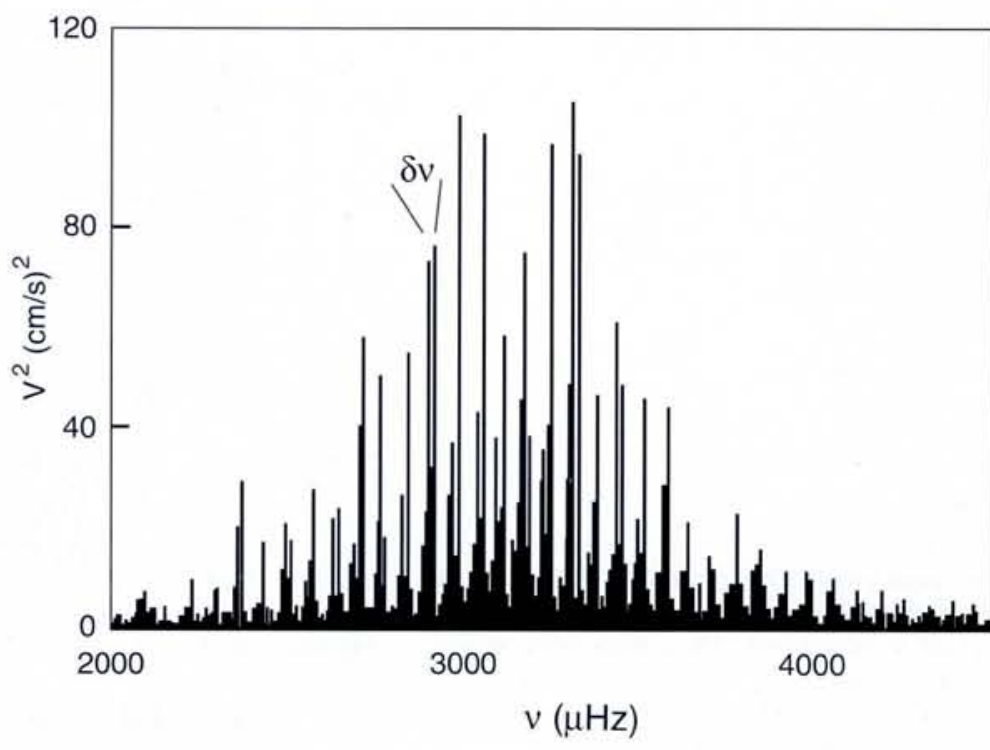

the nature of the oscillations. Owing to their comparatively high frequency, the observed modes are approximately standing sound waves. The speed of sound $c$ increases from the surface towards the centre. Assuming the ideal gas law, $c^{2} \propto T / \mu$ where $T$ is the temperature and the mean molecular weight $\mu$ is defined such that $\mu^{-1}$ is the number of particles per atomic mass unit. The propagation of sound waves resulting from this increase in $c$ is illustrated in Fig. 4: waves which start out from the solar surface obliquely relative to the vertical undergo refraction as they propagate towards the solar interior, in the direction of increasing $c$. With decreasing degree $I$, the initial angle to the vertical decreases, and hence the depth of penetration increases. Radial oscillations $(I=0)$ correspond to vertically propagating rays and hence reach the solar centre; for all other modes there is a zone of avoidance, whose radius $r_{t}$ increases with in-

Thus, only low-degree modes probe the solar core. For such modes, we have

$$
\nu_{n l} \approx \Delta \nu(n+1 / 2+\alpha)+\epsilon_{n l}
$$

where the frequency spacing $\Delta \nu$ is given by $\Delta \nu=\left(2 \int_{0}^{R} \mathrm{~d} r / c\right)^{-1}$, with integration over distance $r$ to the centre up to the surface

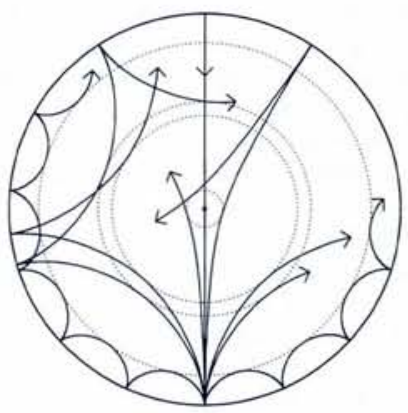

Fig. 4 - Rays illustrating the propagation of acoustic waves in the Sun. The observed oscillations result from interference between such rays. In each case, the dotted line indicates the sphere of avoidance into which the modes do not penetrate. The rays going through the centre correspond to spherically symmetric modes, with $I=0$. radius $R$ of the Sun, is the inverse of the time sound takes to travel across a solar diameter; $\alpha$ is determined by conditions near the surface, and $\epsilon_{n l}$ is a small correction term. If $\epsilon_{n l}$ is neglected, $\nu_{n l} \approx \nu_{n-1 /+2}$ and Eq. 1 predicts a strikingly simple spectrum: the peaks are uniformly spaced, alternating between modes of even and odd degree, with a separation of $\Delta \nu / 2$. This general behaviour is clearly visible in Fig. 3 .

Also visible in the spectrum of Fig. 3 are the effects of the correction term $\epsilon_{n l}$ : there is a small separation

$$
\delta \nu_{n l} \equiv \nu_{n l}-\nu_{n-1} l+2 \approx \epsilon_{n l}-\epsilon_{n-1} 1+2
$$

between peaks that would otherwise coincide. A more careful analysis shows that $\delta \nu_{n l}$ is mainly determined by conditions in the solar core. This is also indicated by the ray paths in Fig. 4: only in and near the zone of avoidance do the paths for $I=2$ differ significantly from those for $I=0$ so the central region dominates the difference $\delta \nu_{n 0}$ between the frequencies of these modes. Furthermore, the uncertainties in the computed frequencies resulting from the poorly known properties of the oscillations near the solar surface depend on frequency but not on degree; hence these uncertainties largely cancel in the difference in Eq. 2. The observed values of $\delta \nu_{n 0}$ thus provide a test of models for solving the neutrino problem.

\section{A caveat}

There is a caveat, however. Frequencies of stellar oscillation are largely determined by the "dynamic" properties of the star, such as the speed of sound and the mass distribution, which in turn depends on the distribution of density $\rho$. It can be shown that, apart from the influence of the superficial layers of the Sun, the frequencies are uniquely determined by the variation of $c$ and $\rho$ with $r$. The temperature $T$ is related to $c$ and $\rho$ through the equation of state, but this also involves the chemical composition: the speed of sound is given approximately by the ratio $T / \mu$, with $\mu$ obviously depending on composition. From the frequencies, information about temperature is therefore only obtained in the combination $T / \mu$. To infer $T$, and hence the expected neutrino flux, the present composition of the solar core must 


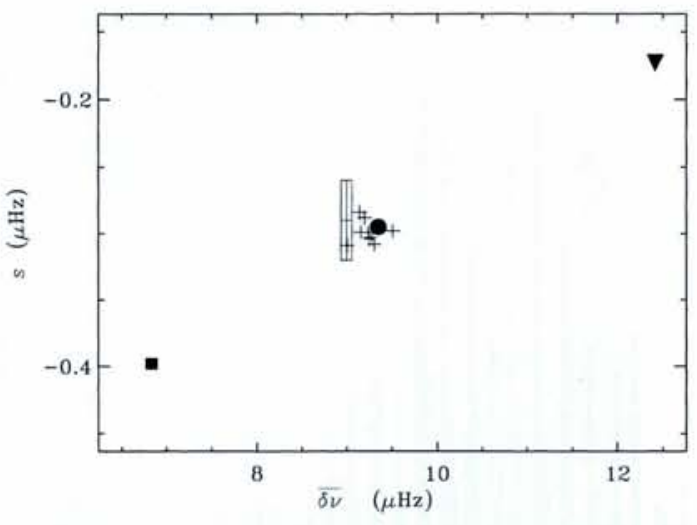

Fig. 5-Parameters characterizing the frequency separation amongst low-degree modes (Eq. 4): $\overline{\delta \nu}$ is the average of the frequency separation $\delta \nu$ (see Fig. 3) and $s$ is the rate of change of $\delta \nu$ with the order of the mode. The error box corresponds to observed values, whereas the points give computed results for the solar models whose neutrino event rates are plotted in Fig. 2 (symbols as in Fig. 2).

be constrained by requiring, for example, that it results from solar evolution under given assumptions. Thus frequency observations cannot by themselves be used to predict the neutrino flux, although they can evidently rule out specific models.

\section{Results}

\section{Standard solar models}

The most accurate measurements of frequencies of low-degree modes have been made using light emission integrated over the solar surface by measuring either the Doppler velocity or the intensity. I shall consider Doppler measurements obtained over several years from a network of observing stations set up by a group in Birmingham [6]. When using the frequency separation $\delta \nu_{n l}$ defined in Eq. 2 for $I=0$, it is convenient to parameterize the dependence of $\delta \nu_{n 0}$ on $n$ through a least-squares fit of the form

$$
\delta \nu_{n 0}=\overline{\delta \nu}+s(n-17)
$$

The results can be represented in a $\overline{\delta \nu}$, $s$ diagram, as shown in Fig. 5 where the error box corresponds to the observed values with error bars of one standard deviation. The points show the results of applying the fit (3) to frequencies computed for a range of "standard" solar models that involve adjusting the physics; their scatter indicates although certainly not in a strict statistical sense - the uncertainty in the computed values. While there are some discrepancies, the standard models give computed results which are generally in good agreement with the observations. Other, independent, observations, notably an extensive set of intensity measurements obtained from the IPHIR experiment on the Phobos Mars probe [7], confirm this conclusion.

\section{Non-standard solar models}

Fig. 5 also gives results for models with a reduced core opacity and with partial mixing of the core (the completely mixed model, whose ${ }^{37} \mathrm{Cl}$ capture rate is consistent with the measurements, falls outside the range of the plot). It is evident that both these models are entirely inconsistent with the oscillation data. More generally, it appears that all specific modifications to solar models proposed to reduce the neutrino flux are ruled out by the frequencies observed.

Although frequency measurements by themselves do not provide definite constraints on the neutrino flux, the failure of the proposed non-standard models to satisfy the test imposed by the frequencies suggests that any such model (e.g., a carefully adjusted combination of WIMPs and mixing)

\section{OTHER APPLICATIONS OF HELIOSEISMOLOGY}

The application to neutrino physics is only a small, though important, corner of helioseismology. Very accurate determinations of solar structure will enable detailed investigation of the properties of the solar plasma, particularly the equation of state and the opacity. This will provide fundamental information about the physics of dense plasmas, under conditions that are out of reach in the laboratory. Furthermore, measurements of the dependence of the frequencies on azimuthal order $m$ allow determination of the solar internal angular velocity as a function of position in the Sun. This is of great importance in understanding the rotation in solar-like stars, from the generally assumed initial state of rapid rotation, and will shed light on the origin of the differential rotation with latitude which is observed on the solar surface. Moreover, interaction between diffe-

rential rotation and the motions in the Sun's outer convection zone is often assumed to be responsible for the cyclic variation of the solar magnetic activity, yet the details, or even the reality, of these $d y$ namo processes are poorly established. Measurements of rotation will clearly help in constraining the models for the generation of the magnetic field. Finally, it seems that helioseismic data will eventually uncover subtle departures from the standard theory of solar evolution, possibly related to hydrodynamic phenomena in the solar interior which can affect the distribution of chemical elements in the Sun. Such effects, when properly understood, would then have to be taken into account in computations of models of other types of stars and might, for example, affect age estimates of stellar clusters based on stellar evolution calculations.

\section{FUTURE PROSPECTS}

Observations of both solar neutrinos and solar oscillations will undergo dramatic improvement in the coming decade. Measurements with the $\mathrm{Ga}$ detectors are still at an early stage; the results will become substantially more accurate with the continuing accumulation of data over the next few years. Furthermore, far more detailed data on the electron scattering will come the Super Kamiokande detector which is expected to begin operations in 1996; and the Sudbury Neutrino Observatory in Canada, with a similar planned starting time, may test the MSW hypothesis by detecting the muon- or tau-neutrinos as well.

In addition to the networks of stations for Doppler observations in integrated light, which are still being completed, the Global Oscillations Network Group (GONG) project will establish a network of six helioseismology observatories, allowing nearly continuous observations of oscillations of degree up to about 300 . Even more detailed data, extending to degrees of at least 1000 , will result from the SOI-MDI instrument on the SOHO spacecraft, to be launched in 1995 [Domingo V. \& Schmidt R. Europhys. News 22 (1991) 213]. Other instruments on SOHO will study low- degree modes with substantially improved sensitivity. This may permit detection of so-called $g$-modes, i.e., standing internal gravity waves. Unlike the acoustic modes considered in the text, the $g$-modes have large amplitudes in the solar core and hence would provide precise information about that region.

Additional insight into stellar evolution will result from detailed analyses of other types of variable stars; a very important contribution, particularly in the study of solar-like oscillations, could be made by the STARS satellite being assessed by the European Space Agency (possibly separate to the Phase $A$ studies for the nex medium-sized mission - see p. 76). Comsity and the speed of sound in the entire solar interior with an improved understanding of stellar evolution, should allow reliable calculation of the spectrum of neutrinos generated in the solar core. The Sun will then essentially constitute a well-calibrated neutrino source and analysis of the results from next-generation neutrino detectors can be used directly to investigate the properties of the neutrinos, as reflected in their interaction with matter or magnetic fields in the solar interior. bining very precise information about den- 


\section{Conclusions}

The conclusions are striking: although small discrepancies remain, there is generally excellent agreement between the results from observed frequencies and those from frequencies of standard solar models. On the other hand, the proposed non-standard models with low neutrino fluxes are generally inconsistent with the oscillation data. By allowing drastic departures from the standard assumptions it is still possible to construct models that agree with the observations for both neutrinos and the oscillations. Nonetheless, the helioseismic results strongly suggest that the resolution of the neutrino problem lies in the realm of particle physics rather than astrophysics. Future work (see insert) will hopefully resolve this major issue.

The most likely explanation involves departures of the properties of the neutrinos from those normally assumed. It has been suggested that the electron-neutrinos produced in the the solar core are transformed by the Mikheyev, Smirnov and Wolfenstein effect (MSW; see [9] for details) into neutrinos of other types (muon- and tau-) on the way to detectors. Such a transformation is possible if there is a finite mass difference between the three types of neutrinos, or if neutrinos have a magnetic moment. It is possible to choose parameters such that the capture rates predicted for standard solar models agree with the measurements.

The effects are too weak to be detectable over the scale of a laboratory on Earth. One way to study them lies in the analysis of

\section{One of 23 New Research Centres}

The Theoretical Astrophysics Center (TAC) is one of 23 research centres set up in 1993 by a new foundation called Danmarks Grundforskningsfond (The Danish National Research Foundation). They were established in 1991 after an extensive and careful evaluation procedure, each with support for an initial five-year period, following a vote by the Danish parliament to grant additional funding for basic research. The centres cover a broad range of topics in the humanities and the natural sciences, and are in most cases associated with one or several existing university institutes.

Apart from TAC, the physical sciences are represented by several centres including the Aarhus Centre for Advanced Physics, based on the ASTRID storage ring which aims to study basic properties of matter and its interaction with radiation, and the Centre for Atomic Material Physics which will make experimental and theoretical investigations of the properties of solid materials, including the growth of atomic layers on surfaces.

TAC is located at the The Niels Bohr Institute for Astrophysics, Physics and Geophysics, Copenhagen, and at the Institute of Physics and Astronomy, Aarhus, with Professor Igor Novikov, Copenhagen, as Director. It aims to concentrate on studies of the origin of largescale structures in the Universe, the formation and evolution of galaxies, and the structure and oscillations of the Sun and other stars. Although the emphasis will be on theoretical work, close contact will be maintained with related observational activities, both within Denmark and abroad. In particular, scientists at TAC take part in large international observational projects in several areas of astrophysics.

TAC is currently in the process of filling several long-term and post-doctoral positions, and establishing a suitable computing environment. It is expected that the Centre will fully operational this autumn. The goal is a centre of excellence in theoretical astrophysics, working closely with groups around the world, and hence providing a major boost to astrophysics research in Denmark.

solar neutrinos provided the conditions in the solar core, and hence neutrino emission, can be constrained by helioseismology.

[1] Bahcall J.N. \& Pinsonneault M.H., Rev. Mod. Phys. 64 (1992) 885.

[2] Claverie A. et al., Mem. Soc. Astron. Ital. 55 (1984) 63.

[3] Bahcall J.N. \& Bethe H.A., Phys. Rev. D 47 (1993) 1298.
[4] Morrison D.R.O., Int. J. Mod, Phys. D1 (1992) 281. [5] Gough D. O. \& Toomre J., Ann. Rev. Astron. Astrophys. 29 (1991) 627.

[6] Elsworth Y. et al., Nature 347 (1990) 536.

[7] Toutain T. \& Fröhlich C., Astron. Astrophys. 257 (1992) 287.

[8] Christensen-Dalsgaard J., Proffitt C.R. \& Thompson M.J., Astrophys. J. 403 (1993) L75.

[9] Bahcall J.N., Neutrino Astrophysics (Cambridge University Press, 1989).

\section{Ulysses is Proving its Worth}

\begin{abstract}
Rudolf von Steiger from the Physikalisches Institut, Bern University, reports that a complete view of the heliosphere is emerging as the Ulysses spacecraft sweeps across the Sun's poles.
\end{abstract}

\begin{abstract}
Ulysses was launched in 1990 and has reached a heliospheric latitude of $60^{\circ} \mathrm{S}$. It will continue to climb in latitude, reaching $80^{\circ} \mathrm{S}$ this September. There follows a fast passage from south to north, reaching $80^{\circ} \mathrm{N}$ in August 1995 (see Fig 1). Much of the new physics that is being discovered in this first visit to the third dimension of our solar system was reviewed last month at the 28th ESLAB Symposium (Friedrichshafen; 19-24 April 1994) that heralded the start of Ulysses's race across the Sun's south pole. A new view of the heliosphere as a three-dimensional, structured, dynamic entity is beginning to emerge, for which the well-known models of the ecliptic will serve as the boundary condi-
\end{abstract} tions of a more global view.

L. Fisk outlined the questions that need to be answered by Ulysses in order that the future judgement of the mission will be kind. They concern: the overall, three-dimensional structure of the solar wind; cosmic rays (both galactic and anomalous) propagating through the solar system; the properties of the interstellar medium entering the heliosphere, and of the so-called pick-up ions that result from its ionization; the source and the acceleration mechanism of the solar wind in the corona.
Fig. 1 - Ulysses's trajectory as it sweeps across the Sun's poles. The spacecraft scans across a high-speed solar wind stream at increasing latitudes during each rotation of the Sun. This stream (shown issuing from the solar surface) is modelled as a co-rotating interaction zone bounded by shock waves.

\section{Acceleration Mechanisms Confirmed}

Owing to the declining solar activity, the polar coronal holes $(\mathrm{CHs})$ have re-formed at high latitudes. The SWOOPS instrument with S. Bame and J. Phillips as the Principal Investigators (PIS) and the SWICS sensors (PIs: J. Geiss and G. Gloeckler) first detected the low-latitude extension of the south polar $\mathrm{CH}$ as a high-speed solar wind (SW) stream in mid-1992, when Ulysses was at $13^{\circ} \mathrm{S}$. The stream subsequently reappeared every solar rotation (i.e., about every 26 days), alternating with periods of low-speed SW that became shorter and shorter. The observation of these repeated transitions, which arise in periods of low solar activity, was fortuitous as they are ideal for comparing the low-speed, in-ecliptic SW with the high-speed streams from $\mathrm{CHs}$.

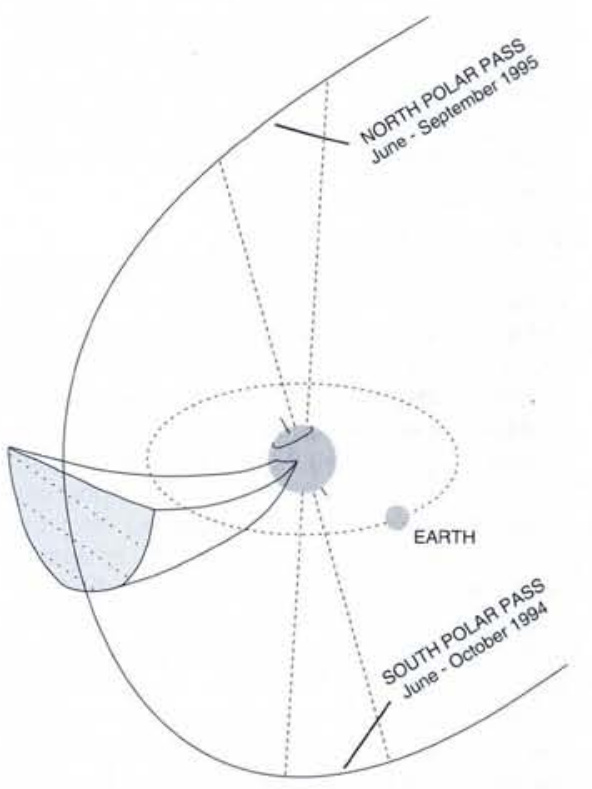

Ulysses has been completely immersed in the high-speed stream from the south polar $\mathrm{CH}$ since mid-1993 when the spacecraft was at $36^{\circ} \mathrm{S}$. At latitudes of this magnitude, the SW's density and pressure are found to be more uniform than those measured in the ecliptic plane by SWOOPS. However, substantial $(\approx 100 \mathrm{~km} / \mathrm{s}$ ) long-term speed variations still persist. Furthermore, SWOOPS finds at high latitudes a latitudinal gradient in the difference between the velocities of protons and alpha-particles which is larger (of 